\title{
Theoretical analysis for calculation of the through thickness effective constants for orthotropic thick filament wound tubes.
}

\begin{abstract}
This paper presents a method for determining the theoretical values for all the elastic constants needed for three-dimensional stresses of angle-ply laminates and filament wound tube from the properties of unidirectional fiber reinforced epoxy resin. The layers were arranged symmetrically about the mid-surface of the laminate. The stress in the longitudinal axis is assumed to be constant throughout the thickness of the laminate. The results for the effective elastic constants versus filament winding angle from $\theta=0^{\circ}$ to $\theta=90^{\circ}$ for glass/epoxy composite are presented. The results show that the through thickness elastic modulus $\mathrm{E} z$ did not change significantly with the winding angle. The $\mathrm{E} x, \mathrm{E} y$, and $\mathrm{G} x \mathrm{y}$ vary significantly with the winding angle of the tube $\theta$. Out of plane shear modulus $\mathrm{G} x \mathrm{x}$ and $\mathrm{G}$ yz also did not vary significantly with the winding angle of the tube $\theta$.
\end{abstract}

Keyword: Orthotropic; Thick filament wound tubes; Through thickness effective constants. 